\title{
Dynamic self-assembly and directed flow of rotating colloids in microchannels
}

\author{
Ingo O. Götze* and Gerhard Gompper ${ }^{\dagger}$ \\ Theoretical Soft Matter and Biophysics, Institute of Complex Systems and Institute for Advanced Simulation, Forschungszentrum Jülich, \\ Jülich D-52425, Germany
}

(Received 24 June 2011; published 19 September 2011; publisher error corrected 23 September 2011)

\begin{abstract}
Nonequilibrium structure formation and dynamics in suspensions of superparamagnetic colloids driven by an external rotating magnetic field are studied by particle-based mesoscale hydrodynamics simulations in confined geometry. We address the fundamental question how the rotation of the colloids about their own axes can be converted into a translational motion by breaking the symmetry of the confining geometry. We study a two-dimensional system of colloids with short-range repulsive interactions, which mimics flow in shallow microchannels. In straight channels, we observe a two-way traffic but-for symmetry reasons-no net transport. However, by keeping some colloids fixed near one of the two walls, net transport can be achieved. This approach allows the control and switchability of the flow in complex microchannel networks. A minimal geometry that fulfills the requirement of broken symmetry are ring channels. We determine the translational velocity of the spinning colloids and study its dependence on the channel width for various median radii. We conclude that spinning colloids present a promising alternative for flow generation and control in microfluidic devices.
\end{abstract}

DOI: 10.1103/PhysRevE.84.031404

PACS number(s): 83.80.Hj, 83.10.Rs, 47.61.-k, 47.57.-s

\section{INTRODUCTION}

Externally actuated and self-propelled micro- and nanorotators show an intriguing variety of nonequilibrium structure formation and dynamics. Examples of such systems include superparamagnetic colloidal particles in a rotating magnetic field [1-4], dipolar colloids in a rotating electric field [5], colloidal dimers rotated by laser tweezers [6], and biological swimmers such as Volvox algae [7]. The collective behavior of rotator suspensions is governed by hydrodynamic interactions. In bulk fluids, two Volvox algae, spinning due to beating cilia on their surfaces, form stable bound states [7]; several spinning magnetic disks at the liquid-air interface spontaneously assemble in rotating clusters with a hexagonal crystal structure [1,2]; and suspensions of rotators at sufficiently large volume fraction show an enhanced translational diffusion due to their spinning motion [8]. In confined geometry, spinning colloids also show interesting features; for example, spinning colloids placed asymmetrically in a microfluidic channel act as micropumps $[3,4,6]$.

Microfluidic devices have recently attracted much interest due to their vast variety of applications in medical diagnosis and chemical synthesis [9]. The main advantages of microfluidic systems are that they require only small fluid volumes (sample as well as reagents), their high efficiency due to the good surface to volume ratio, fast response times because of short diffusion distances, and that they allow the application at the point of need. An integral part of these systems are micropumps. Recent experimental designs include colloidal particles that are actuated by optical tweezers $[6,10]$ and selfassembling superparamagnetic colloids in an external rotating magnetic field [3]. An advantage of spinning colloids is that they allow the massively parallel operation of micropumps.

Simulations can help to gain insight into the flow properties of complex fluids and are therefore important for

\footnotetext{
*i.goetze@fz-juelich.de

${ }^{\dagger}$ g.gompper@fz-juelich.de
}

understanding the mechanisms of flow generation and dynamical properties in microfluidic devices. Hydrodynamic interactions and thermal fluctuations often play an essential role in soft matter systems. However, the gap between the mesoscopic length and time scales of the macromolecules or colloids and the microscopic scales of the solvent prohibits a full molecular dynamics (MD) treatment on the atomistic level. Therefore, a mesoscale simulation technique is required, which is sufficiently efficient to be tractable, but at the same time captures the relevant physical behavior. We employ here the multi-particle collision dynamics (MPC) approach, which naturally includes both hydrodynamics and thermal motion [11-13].

In order to understand the basic physical mechanisms of flow generation by rotating colloids, it is important to reduce the geometry of the problem to its essential features. A key prerequisite for converting rotational into a translational motion is symmetry breaking in the confining geometry. This can be achieved, for example, by curvature of a microchannel [14] or by asymetric boundary conditions in a linear channel $[15,16]$. Since the height of microchannels is typically much smaller than their width, and the rotators in Ref. [3] are selfassembled disklike clusters of superparamagnetic colloidal particles, we focus on two-dimensional (2D) model systems; see Fig. 1. Discs that are free to move are embedded in the fluid, with each disk exposed to the same external torque.

In the last 160 years, there have been several different approaches to deal with the problem of the viscous flow around cylinders (or disks in two dimensions). In contrast to spheres, even for the simple case of flow around a single infinitely extended cylinder, there exists no solution of the low-Reynolds-number Navier-Stokes equations (Stokes's paradox) [17-19]. However, these difficulties only apply to unbounded fluid domains. In Ref. [20,21], the flow induced by a macroscopic rotating cylinder with fixed axis placed at an off-center position in a planar channel has been studied by finite-element methods, and in Ref. [22], the flow past a single freely rotating cylinder was studied numerically. Passively rotating cylinders between actively rotating 


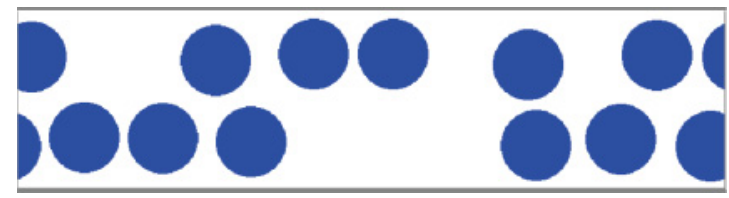

FIG. 1. (Color online) Simulation snapshot of 12 spinning colloids (diameter $\sigma$ in a straight channel of width $D=2.5 \sigma$ and length $10 \sigma$ with periodic boundary conditions in the lateral direction).

confining cylinders have been investigated in Ref. [23] using multipole-accelerated boundary-element methods. Rotating cylinders and rotlets (infinitesimal cylinders) with fixed axis have been studied in Refs. [24,25]. However, all these studies neglect thermal fluctuations, which become increasingly important with advancing miniaturization of microfluidic devices.

A well-established mesoscale hydrodynamics simulation technique that naturally includes thermal fluctuations is multiparticle collision dynamics (MPC) [11]. This particlebased method has been applied to various systems such as colloids [26-29], polymers [30-36], membranes, vesicles and red blood cells [37,38], ternary amphiphilic fluids [39], and chemical reaction systems [40]. An overview can be found in the reviews of Refs. [12,13]. The algorithm is computationally very efficient and is constructed such that mass, energy, and translational momentum are locally conserved, which is essential for correct hydrodynamic behavior to emerge. Hybrid simulations combining an MPC fluid with MD for the solute particles as well as coupling to moving boundaries are easily possible. We employ here an angular momentum conserving version [41], denoted $\mathrm{MPC}-\mathrm{AT}+a$, for which it has been shown that it yields the correct forces and flow fields for two rotating eccentric cylinders [42] (which is not the case for the standard version, denoted stochastic rotation dynamics).

\section{SIMULATION METHOD}

\section{A. Multiparticle collision dynamics (MPC)}

In MPC simulations, the fluid is represented by $N$ point particles of mass $m$ that undergo two alternating steps. In the streaming step, the particles propagate freely, i.e., their positions $\mathbf{r}_{i}$ are updated according to $\mathbf{r}_{i}(t+\Delta t)=\mathbf{r}_{i}(t)+$ $\mathbf{v}_{i} \Delta t$, where $\Delta t$ is the time interval between collisions and $\mathbf{v}_{i}$ are the particle velocities. Subsequently, the particles are sorted into cubic cells of lattice constant $a$, hereafter referred to as collision cells. This superimposed lattice is randomly shifted in each step to ensure Galilean invariance [43]. By assigning the particles new velocities, the collision step then mimics the simultaneous interaction of all particles within each collision cell. Here linear momentum is conserved in each cell, in order to obtain correct hydrodynamic behavior. Since the standard MPC collision algorithm (stochastic rotation dynamics [11]) does not conserve angular momentum, which has been shown to be essential when torques acting on rotating colloids are considered [42], we employ an angular momentum conserving variant $(\mathrm{MPC}-\mathrm{AT}+a)$, where the velocities of particles are updated by $[41,42]$

$$
\begin{aligned}
\mathbf{v}_{i}^{\prime}= & \mathbf{v}_{\mathrm{c}}^{\mathrm{G}}+\mathbf{v}_{i}^{\mathrm{ran}}-\sum_{j \in \mathrm{cell}} \mathbf{v}_{j}^{\mathrm{ran}} / N_{\mathrm{c}} \\
& +m \Pi^{-1} \sum_{j \in \text { cell }}\left[\mathbf{r}_{j, \mathrm{c}} \times\left(\mathbf{v}_{j}-\mathbf{v}_{j}^{\mathrm{ran}}\right)\right] \times \mathbf{r}_{i, \mathrm{c}},
\end{aligned}
$$

where $\Pi$ is the moment-of-inertia tensor of the particles in the cell. The relative position is $\mathbf{r}_{i, \mathrm{c}}=\mathbf{r}_{i}-\mathbf{r}_{\mathrm{c}}^{\mathrm{G}}$, where $\mathbf{r}_{\mathrm{c}}^{\mathrm{G}}$ is the center of mass of the particles in the cell. This collision method serves at the same time as a thermostat.

\section{B. Boundary conditions}

The colloids are treated as (2D) spherical, movable boundaries of diameter $\sigma$ that are impenetrable for the fluid particles. In the case of the ring channels, the colloids and fluid are confined between two fixed concentric cylinders with the inner and outer wall radii $R_{i}$ and $R_{o}$, respectively. The linear channels are parallel walls with distance $D$ and periodic boundary conditions in the direction of the channel. The fluidwall interaction and the fluid-colloid interaction are modeled as hard interactions, i.e., the potential is $V=\infty$ inside walls and colloids, and $V=0$ otherwise. In order to account for the microscopic roughness of the confining walls and the surface of the colloids, stick (no-slip) boundary conditions are used. In the streaming step, this is achieved by scattering the particles with a bounce-back rule on surfaces: $\mathbf{v}^{\prime}=-\mathbf{v}+2 \mathbf{v}_{\text {sur }}$, where $\mathbf{v}_{\text {sur }}$ is the local velocity of the surface, and $\mathbf{v}$ and $\mathbf{v}^{\prime}$ are the old and new particle velocities, respectively. In the collision step, virtual particles are required to reduce the slip on the surfaces [44]. Here the virtual particles are distributed randomly within layers of width $\sqrt{2} a$ along all surfaces before each collision step, as described in Refs. [14,42]. Their velocities are chosen from a Maxwell-Boltzmann distribution with zero mean velocity. For the colloids, the local velocity $\mathbf{V}_{j}+\left(\Omega_{j} \times \hat{\mathbf{r}}_{i}^{\text {virt }}\right) \sigma_{\text {col }} / 2$ is added, where $\mathbf{V}_{j}$ and $\Omega_{j}$ are the velocity and angular velocity of the corresponding colloid $j$, respectively, and $\hat{\mathbf{r}}_{i}^{\text {virt }}$ is the normalized relative position vector of virtual particle $i$ with respect to the center of colloid $j$. Consequently the forces and the torques exerted by the fluid on the colloids consist of a kinetic and a collisional contribution.

During the streaming step, the change of the linear momentum and the angular momentum of all fluid particles that collide with colloid $j$ are accumulated, i.e., $\Delta \mathbf{P}_{j}^{\mathrm{kin}}=$ $\sum m\left(\mathbf{v}_{i}-\mathbf{v}_{i}^{\prime}\right)$ and $\Delta \mathbf{L}_{j}^{\mathrm{kin}}=\sum m \mathbf{r}_{i}^{*} \times\left(\mathbf{v}_{i}-\mathbf{v}_{i}^{\prime}\right)$, where $\mathbf{r}_{i}^{*}$ is the relative position of the collision of particle $i$ with respect to the colloid's center. The linear and angular velocities of the colloids are then updated according to $\mathbf{V}_{j}^{\prime}=\mathbf{V}_{j}+\Delta \mathbf{P}_{j}^{\mathrm{kin}} / M$ and $\Omega_{j}^{\prime}=\Omega_{j}+\Delta \mathbf{L}_{j}^{\mathrm{kin}} / I$, where $M$ and $I$ are the mass and the moment of inertia of the colloid, respectively. In the collision step, momentum is exchanged in cells that contain virtual as well as real particles at the same time, so that $\Delta \mathbf{P}_{j}^{\text {coll }}=$ $\sum m\left(\mathbf{v}_{\text {virt }}^{\prime}-\mathbf{v}_{\text {virt }}\right)$ and $\Delta \mathbf{L}_{j}^{\text {coll }}=\sum m \mathbf{r}_{\text {virt }} \times\left(\mathbf{v}_{\text {virt }}^{\prime}-\mathbf{v}_{\text {virt }}\right)$. The new linear and angular velocities of the colloids at the end of the time step are then given by $\mathbf{V}_{j}^{\prime}=\mathbf{V}_{j}+\Delta \mathbf{P}_{j}^{\text {coll }} / M$ and $\Omega_{j}^{\prime}=\Omega_{j}+\Delta \mathbf{L}_{j}^{\text {coll }} / I$.

Since the hydrodynamics are only correctly resolved on length scales larger than the collision cell size $a$, we add a short-range Lennard-Jones repulsion to the excluded volume 
in order to avoid direct contact of colloids with the walls and/or with other colloids:

$$
V^{\mathrm{LJ}}(s)=4 \epsilon\left[\left(\frac{\delta_{\mathrm{LJ}}}{s}\right)^{12}-\left(\frac{\delta_{\mathrm{LJ}}}{s}\right)^{6}\right]+\epsilon .
$$

The colloid-colloid interaction is then given by

$$
V\left(r_{i j}\right)= \begin{cases}\infty, & r_{i j}<\sigma, \\ V^{\mathrm{LJ}}\left(r_{i j}-\sigma\right), & \sigma<r_{i j}<r_{\text {cut }}, \\ 0, & \text { else, }\end{cases}
$$

where $r_{i j}$ is the colloid-colloid distance, $r_{\text {cut }}$ is the cutoff radius, and $\epsilon$ and $\delta_{\mathrm{LJ}}$ set the potential strength and range, respectively. Similarly, the colloid wall interactions are

$$
V(r)= \begin{cases}\infty, & r<\tilde{R}_{i}, \\ V^{\mathrm{LJ}}\left(r-\frac{\sigma}{2}-R_{i}\right), & \tilde{R}_{i}<r<\tilde{R}_{i}+r_{\mathrm{cut}}, \\ 0, & r>\tilde{R}_{i}+r_{\text {cut }},\end{cases}
$$

with $\tilde{R}_{i}=R_{i}+\sigma / 2$ for the inner wall, and

$$
V(r)= \begin{cases}\infty, & r>\tilde{R}_{o}, \\ V^{\mathrm{LJ}}\left(r+\frac{\sigma}{2}-R_{o}\right), & \tilde{R}_{o}-r_{\text {cut }}<r<\tilde{R}_{o}, \\ 0, & r<\tilde{R}_{o}-r_{\text {cut }},\end{cases}
$$

with $\tilde{R}_{o}=R_{o}-\sigma / 2$ for the outer wall. The colloid-wall interactions for the linear channel are defined analogously. The positions of the colloids are updated by performing several MD steps between the MPC collisions, using the velocity-Verlet algorithm.

\section{Parameters}

We simulate 2D flows. The simulation data are displayed with the units of length $\sigma$ and time $\tau=a \sqrt{m / k_{\mathrm{B}} T}$, respectively. We use $\Delta t / \tau=0.02$ and the average number of fluid particles per collision cell is $n=10$. For these parameters, the collisional contribution to the viscosity $\eta$ dominates over the kinetic contribution; the analytical expressions for the viscosity of MPC-AT $+a$ derived in Ref. [45] yield $\eta=$ $18 \sqrt{m k_{\mathrm{B}} T} / a^{2}$. The geometry of the ring channel (annulus) is characterized by its median radius $R$ and its width $D$, so that $R_{i}=R-D / 2$ and $R_{o}=R+D / 2$. While varying $R$ and $D$, we keep the colloid line density $N_{\text {col }} /(2 \pi R)$ fixed at $1 /(4 \pi) a^{-1}$.

The finite range of the multiparticle interaction in the collision step sets limitations to the validity of the hydrodynamics at short length scales. Therefore, colloid sizes much larger than the collision-cell size $a$ are required. Here we use $\sigma=10 a$. Furthermore, we chose $\epsilon=k_{B} T, \delta_{\mathrm{LJ}}=a$ and $r_{\text {cut }}=2^{1 / 6} a$ for the thin soft-repulsion layer in addition to the hard interaction. The mass density of the colloids is chosen to be the same as the fluid mass density; i.e., the mass of the colloids is given by $M=m n \pi \sigma^{2} / 4 \approx 785 m$ for our choice of parameters. Motivated by the experiments on superparamagnetic particles [3], we apply a constant external torque to the colloids. An external torque results in an average angular velocity $\Omega_{\infty}=L_{\text {ext }} /\left(\eta \pi \sigma^{2}\right)$ in an infinitely extended fluid, where $\eta$ is the viscosity of the fluid. In order to avoid high surface velocities $\mathbf{v}_{\text {sur }}=\mathbf{V}+\Omega \sigma / 2$, we choose the torque such that $\Omega_{\infty} \sigma / 2<0.1 a \tau^{-1}$. This corresponds to a small
Reynolds number $\operatorname{Re}=\Omega_{\infty} \sigma^{2} \rho /(2 \eta)<0.73$ for the largest torques considered.

The translational diffusion constant $D_{t}$ for a colloid is measured without external torque in a wide ring channel of diameter $D=2.5 \sigma$. The Péclet number Pe for the rotating colloids is then given by $\mathrm{Pe}=\sigma v / D_{t}$, where $v$ is the average tangential velocity. The velocity and thus Pe depend on the considered geometry and on the applied external torque. In order to study the influence of thermal fluctuations, we use three different torques to obtain different Péclet numbers. For the largest torque, $L_{\text {ext }, 0}=150 k_{B} T$, the Péclet number varies between $\mathrm{Pe}=\mathcal{O}(10)$ (narrowest channel) and $\mathrm{Pe}=\mathcal{O}\left(10^{2}\right)$ (widest channel). For the widest channel, the Péclet number varies linearly with the colloid torque, with $\mathrm{Pe}=\mathcal{O}(10)$ for the smallest torque $L_{\text {ext }, 0} / 9=16.7 k_{B} T$. If not mentioned otherwise, the torque $L_{\text {ext, } 0}$ is employed.

\section{RESULTS FOR LINEAR CHANNELS}

\section{A. Linear channels with freely moving colloids}

We first consider spinning colloids in straight channels. For a rotating cylinder close to a single wall, the resulting hydrodynamic forces vanish $[46,47]$. However, in the presence of a second wall, the cylinder experiences a net force, unless it is centered between the walls. Micropumps based on this principle have been studied experimentally [48] and numerically [20,21]. Moreover, pairs of corotating cylinders in an unbounded viscous fluid mutually exert forces on each other that are perpendicular to their connecting line at leading-order of approximation [49], causing the pairs to circulate about each other.

Pairs of colloids close to opposing walls enhance the hydrodynamic forces that would act on individual colloids, leading to accelerated opposite motions along the walls. In straight channels that are wide enough to allow colloids to pass each other, this causes a two-way-traffic-like behavior. At finite colloid density, the colloids tend to stay close to the walls at distance $\sigma / 2+\delta_{\mathrm{LJ}}$, as shown by the probability density in Fig. 2(a) for a wall separation $D=3 \sigma$. With increasing colloid density, colloids push each other hydrodynamically toward the walls, and the peak height in the density distribution increases. The two small additional peaks in Fig. 2(a) at a distance $3 / 2 \sigma+2 \delta_{\mathrm{LJ}}$ from the walls arise from colloids that circumnavigate other colloids close to the opposite walls, i.e., at a distance of approximately $\sigma+\delta_{\mathrm{LJ}}$ from the colloids at the walls.

When the colloids stay close to the walls, an unhindered two-way traffic is facilitated. However, due to thermal fluctuations colloids can change lanes and thus the direction of motion. For lower Pe, the peaks in the colloid density distribution are less pronounced, since they are smeared out by the thermal motion; see Fig. 2(b).

The density peaks close to the walls for large area fractions $\Phi$ of spinning colloids in Figs. 2(a) and 2(b) are much more pronounced than the layering found in corresponding simulations at the same colloid area fractions at thermal equilibrium [see Fig. 2(c)]. In contrast, for very low colloid area fractions and large torques, a maximum in the channel center is observed; see Fig. 2(a); here the local colloid density 

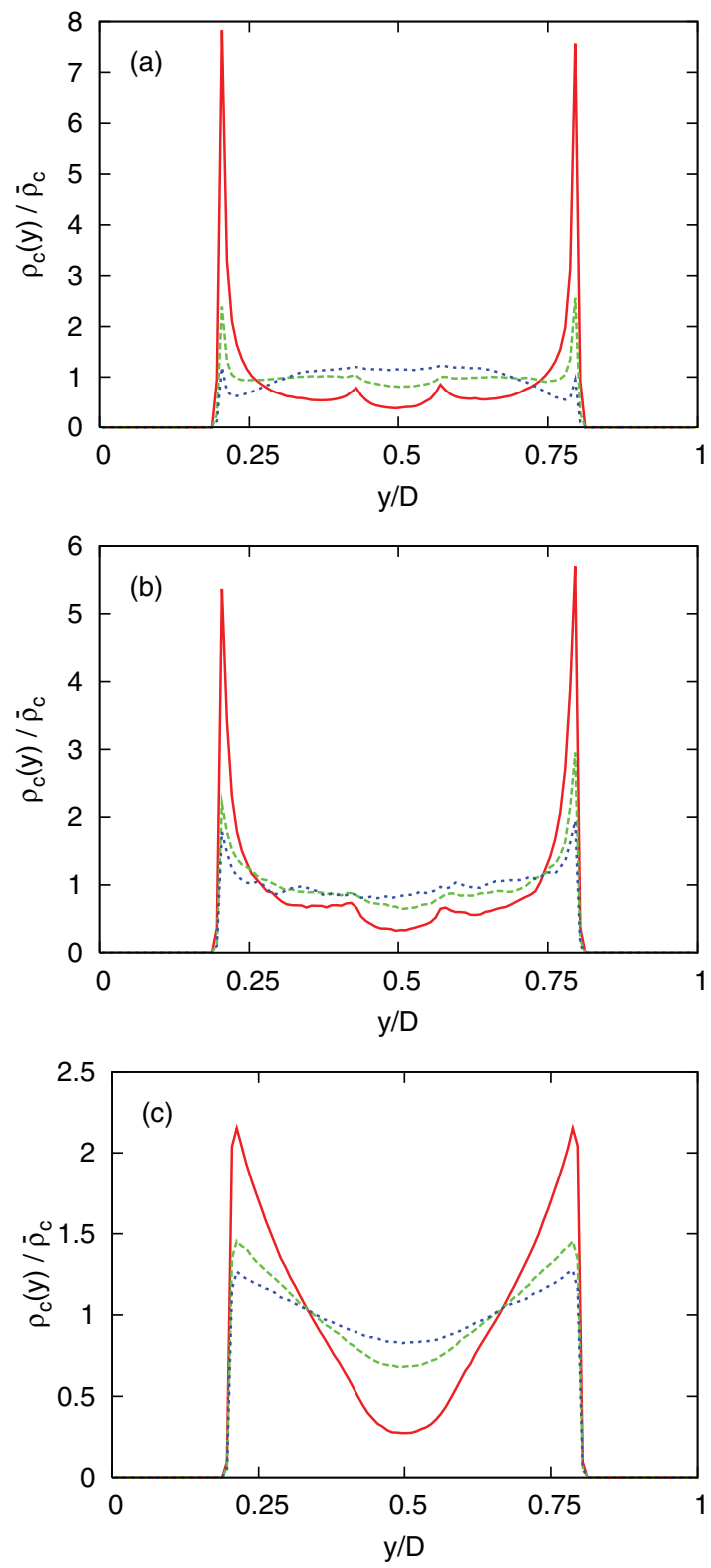

FIG. 2. (Color online) Normalized density profiles of spinning colloids across a linear channel with $D=3.0 \sigma$ at different colloid area fractions $\Phi=0.314$ (red solid line), $\Phi=0.157$ (green dashed line), and $\Phi=0.105$ (blue dotted line). (a) High Pe with torque $L_{0}^{\text {ext }}$, (b) low Pe with torque $L_{0}^{\text {ext }} / 3$, and (c) at equilibrium.

in the center is higher than in thermal equilibrium. Since the Reynolds number is of order unity in this case $(\operatorname{Re} \simeq 0.7)$, we believe that this is related to the Segre-Silberberg effect [50] of particle migration in Poiseuille flow due to inertia.

Colloid velocity profiles for different colloid densities are shown in Fig. 3. For small density, the colloid velocity profile is nearly linear, reflecting the hydrodynamic force on a single rotating colloid between two walls. The small dip at the surface is probably due to a slowing down of the rotational motion as a result of increased friction near the wall. With increasing colloid density, the central part of the profile remains unchanged, while the velocity within the lanes levels off, because all colloids within a lane have to move with the same velocity. Furthermore, since we apply a constant torque

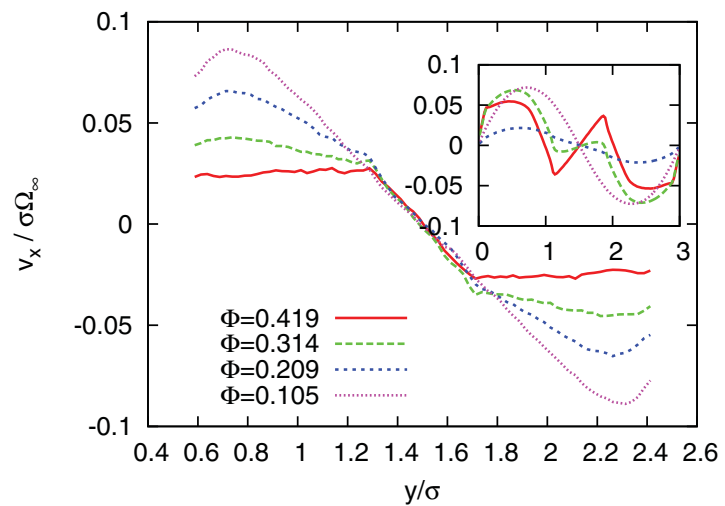

FIG. 3. (Color online) Colloid velocity profiles for a linear channel with $D=3.0 \sigma$ for different colloid area fractions $\Phi$, as indicated, and the corresponding fluid velocity profiles (inset).

to the colloids, they rotate slower and thus also move slower at larger densities. The corresponding fluid velocity profiles are displayed in the inset of Fig. 3. The complex behavior of the fluid velocity profile is a result of the combination of colloid motion pressing the liquid column ahead plus the surface velocity due to the colloid rotation, which are in turn affected by the velocity field caused by the colloids at the opposite wall.

The average colloid velocity per half channel exhibits a maximum at finite colloid densities, as we have shown in Ref. [14]. This behavior can be understood as follows. In the limit of vanishing colloid densities, the colloid velocity per half channel increases with increasing density, because it is more likely to find colloids near the walls where the main thrust is generated. Moreover, colloids near opposite walls propel each other. On the other hand, if the colloid density is to high, they hinder each other, and since the external torque is constant, the rotational velocity decreases. Thus, at high colloid densities, the average colloid velocity per half channel decreases with increasing density.

Although the colloid motion can be described as a twoway traffic, colloids are still able to change lanes due to thermal fluctuations. It is therefore interesting to investigate the dependence of the time a colloid remains in one lane on the Péclet number. In order to eliminate short-time fluctuations near the channel center line, we divide the channel into two lanes near the walls that are separated by a region of width $d$ (see Fig. 4). We define the time a colloid stays in one lane to be the time between the first entrance into this lane until the first entrance into the lane at the opposite wall. The distribution of times between lane changes is shown in Fig. 5 for $\mathrm{Pe}_{0}$ and $\mathrm{Pe}_{0} / 9$. In the limit $\mathrm{Pe}=0$, this would correspond to the

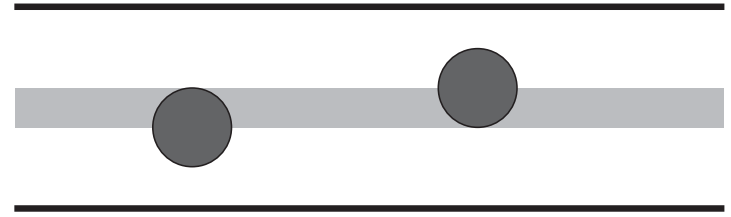

FIG. 4. Illustration of the definition for the two lanes in a straight channel separated by a strip of width $d=\sigma / 2$. 


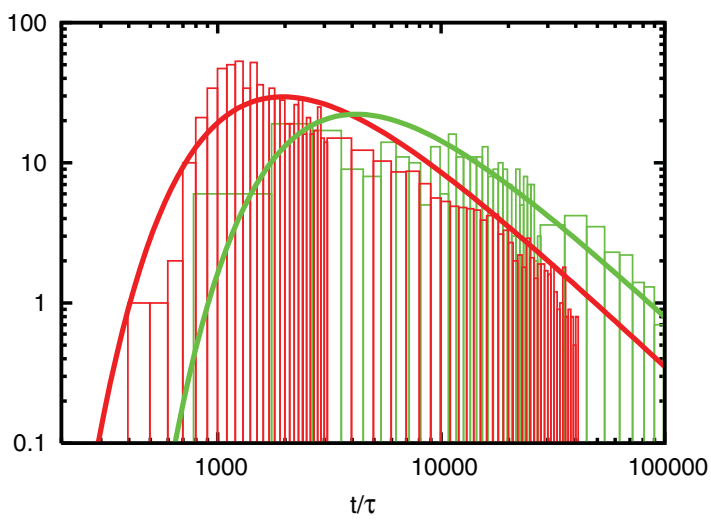

FIG. 5. (Color online) Histogram (not normalized) over the time between two lane changes in a linear channel for Péclet numbers $\mathrm{Pe}_{0}$ (dark gray/red columns) and $\mathrm{Pe}_{0} / 9$ (light gray/green columns). The channel width is $D=2.5 \sigma$ and the colloid area fraction $\Phi=0.25$. The solid lines are fits (by eye) to Eq. (6).

first-passage time of a random walk in one dimension, where the probability is given by [51]

$$
P(t)=\frac{d}{\sqrt{4 \pi D_{0} t^{3}}} e^{-d^{2} / 4 D_{0} t}
$$

in the absence of confining walls. With walls, the firstpassage-time distribution is modified for longer times, because long excursions away from the dividing strip are suppressed; however, this effect is not important in our analysis. Figure 5 shows that Eq. (6) describes the histograms obtained from the simulations reasonably well. Therefore, a fit to Eq. (6) provides an estimate of the effective diffusion constant $D_{\text {eff }}(\mathrm{Pe})$ of the colloids. We obtain $D_{\text {eff }}=0.0010 a^{2} / \tau$ for $\mathrm{Pe}_{0} / 9$, and $D_{\text {eff }}=$ $0.0021 a^{2} / \tau$ for $\mathrm{Pe}_{0}$. Thus, the effective diffusion coefficient increases weakly with increasing Péclet number. For low Pe, the behavior is dominated by equilibrium thermal diffusion, and the value of the diffusion coefficient is consistent with the equilibrium diffusion coefficient for the same channel width. The increase of the effective diffusion coefficient for high Pe can be attributed to the circling motion when two colloids come close to each other, because hydrodynamic interaction strengthen with increasing spinning frequency. It is important to emphasize that the distance a colloid travels within each lane strongly increases with increasing Péclet number, because the increased velocity strongly overcompensates the increased diffusion constant.

\section{B. Linear channels with a periodic array of fixed colloids near the wall}

Net transport in a linear channel requires symmetry breaking. This can be achieved by fixing the positions of a linear periodic array of colloids near one of the two walls; see Fig. 6. We start by considering a single fixed colloid in the periodically repeated channel segment. The fixed colloid is allowed to spin; however, the qualitative behavior is very similar if the fixed colloid is not allowed to spin, because due to the vicinity of the wall, its spinning frequency is much smaller than for free colloids. Since one of the two lanes is now blocked, there is a net flow in the open lane. Alternatively, this situation can

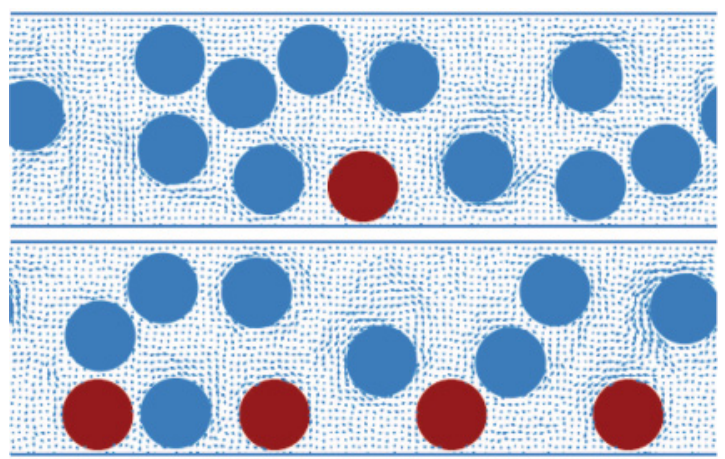

FIG. 6. (Color online) Simulation snapshots of spinning colloids in a channel, for which some colloids (red/dark gray) are fixed in position near the lower side of the channel. (Top) 1 of 12 colloids fixed; (bottom) 4 of 12 colloids fixed. All other colloids (blue/light gray) are free to move. The flow field is indicated by arrows. The channel width is $D=3.0 \sigma$, the channel length is $10 \sigma$, and the colloid area fraction is $\Phi=0.314$. A movie of the colloid motion in a channel with a fixed colloid is provided in the supplemental material [52].

be viewed as a narrower channel in which colloids can move freely, but with no-slip boundary conditions on one side (at the hard wall), and some slip on the other side (the wall with the fixed colloids).

We vary the total number of colloids, while keeping the colloid area fraction constant (at $\Phi=0.314$ ). The colloid velocity is averaged over all colloids (including the fixed one), since this quantity is proportional to the colloid transport rate. The results are shown in Fig. 7 as a function of the total number of colloids (in a periodic segment) for three different channel widths. In all three cases, the velocity decreases with increasing ratio of the total colloid number to the number of fixed ones. This can be understood as follows. With increasing colloid number, the length of the periodic channel segment increases, so that the line density of fixed colloids decreases; therefore, an increasing part of the channel segment is unaffected by the fixed colloid and does not contribute

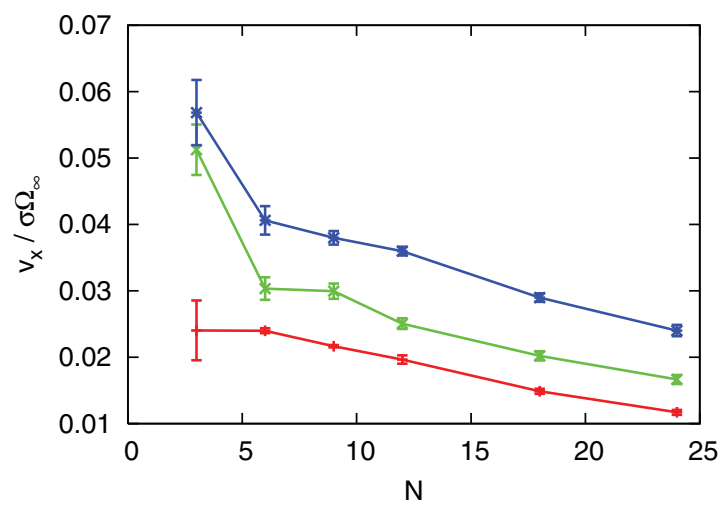

FIG. 7. (Color online) Average colloid velocity in a linear channel with one fixed colloid and $(N-1)$ freely moving colloids as a function of the total number $N$ of colloids, for $D=2.5 \sigma$ (red, bottom), $D=3.0 \sigma$ (green, middle), $D=3.5 \sigma$ (blue, top). The colloid line density is kept constant, with corresponding colloid area fractions of $\Phi=0.377$ for $D=2.5 \sigma, \Phi=0.314$ for $D=3.0 \sigma$, and $\Phi=0.269$ for $D=3.5 \sigma$. 
to the net transport. Furthermore, the fixed colloid reduces the half-channel velocity in the same lane. Thus, the optimal condition for large translational transport should be to fix about $30 \%$ to $50 \%$ of all colloids near one wall.

The corresponding fluid velocity profiles are shown in Fig. 8(a). They demonstrate that the velocity profiles are nearly symmetric for large $N$ (and thus long channel segments), which indicates very small net transport, but pronouncedly asymmetric for $N=3$. In order to estimate finite-size effects, we compare a system with three colloids (one fixed) with a system with 12 colloids (four fixed; see Fig. 6(b)); the corresponding velocity profiles are shown in Fig. 8(b). The comparison shows that the effect of the periodic images in the system with only three colloids in a periodic box leads to an overestimation of the net fluid flow. This effect is responsible for the sharp increase of the transport velocity in Fig. 7 for $N=3$. The finite-size effects are less pronounced for larger $N$.

The possibility of fixing the position of some selected colloids in a channel near the walls, and to change the location of these fixed positions on a time scale much longer than the typical spinning time, allows us to vary the effective boundary conditions in microchannels. Thereby, the flow velocity and the flow direction in simple channels as well as in complex microchannel networks can be controlled. As an example for
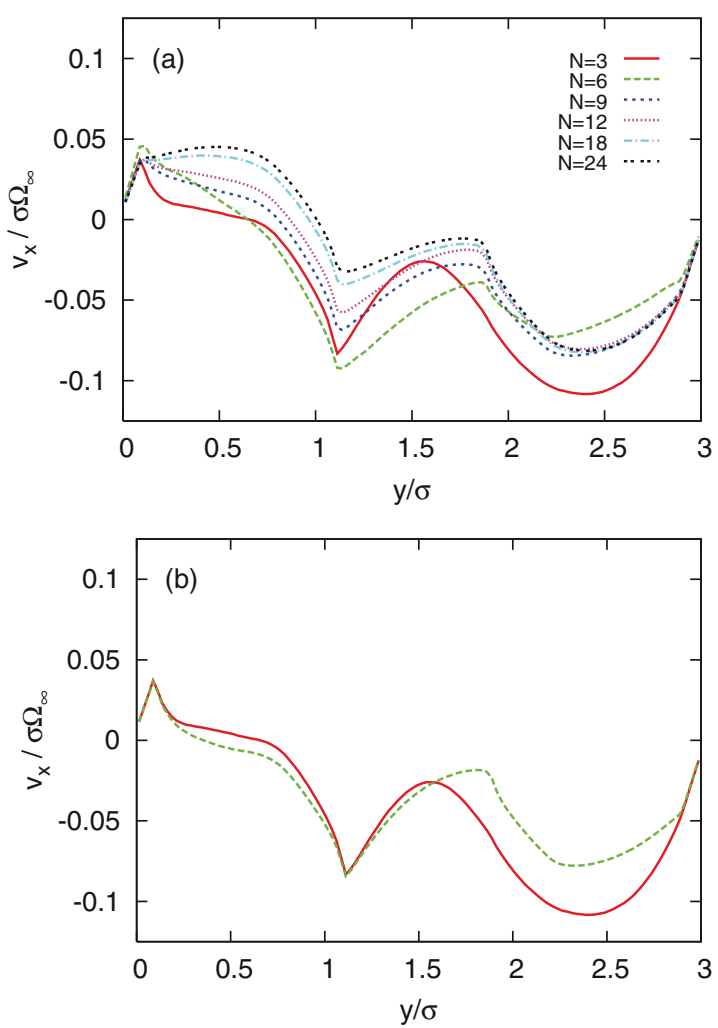

FIG. 8. (Color online) Fluid velocity profiles for channels with fixed colloid near one wall. (a) Variation of the density of fixed colloids, with one colloidal particle fixed, and various total numbers of colloids, as indicated. (b) Variation of the periodic tube segment, with three colloids of which one is fixed (red solid line), and 12 colloids, of which four are fixed (green dashed line). The total colloid density is constant, $\Phi=0.314$. The channel width is $D=3.0 \sigma$.

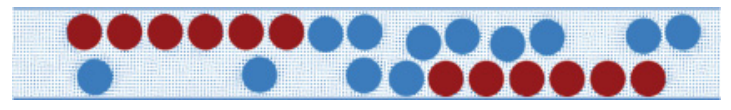

FIG. 9. (Color online) Simulation snapshot for a channel with alternating pumping direction. Six colloids (red/dark gray) are fixed in position at the upper wall on the left-hand side of the channel, and another six at the lower wall on the right-hand side. All other colloids (blue/light gray) are free to move. The channel width is $D=2.5 \sigma$.

a channel with more complex boundary conditions, a linear channel with opposite pumping directions in the left and right sections of a channel segment is shown in Fig. 9. Here the colloids are pumped toward the middle of the channel segment. This give rise to a aggregation of colloids, which is reflected in a peak in the colloid density along this channel, as shown in Fig. 10. However, there is a fluid backflow in the gap caused by the fixed colloids (as indicated in Fig. 11), which makes colloid motion in such a blocked configuration very slow.

\section{RESULTS FOR RING CHANNELS}

\section{A. Tangential force generated by colloid rotation in ring channels}

The forces due to pressure inhomogeneities generated by a rotating colloid close to a concave wall dominate over the viscous forces and thus give rise to a motion of the colloid opposite to the direction expected for a rolling motion $[14,42,46]$ - in contrast to a planar wall, where these two contributions exactly compensate (in two dimensions). However, in an annular region, the velocity field is strongly affected by the presence of the second wall, and the problem cannot be considered as a superposition of the effect of the two walls separately.

Figure 12 shows the tangential component of the force acting on a single rotating colloid as a function of the radial position $r$ of the fixed axis of rotation in the channel. Close to the outer wall, the colloid experiences a force pushing it in a counterclockwise direction in the annulus. Near the inner wall, the sign of the force reverses. This behavior is similar as for linear channels. However, in ring channels the forces near the inner and outer walls differ in magnitude, with a higher magnitude near the outer wall. For $D=2.0 \sigma$ and $2.5 \sigma$, the

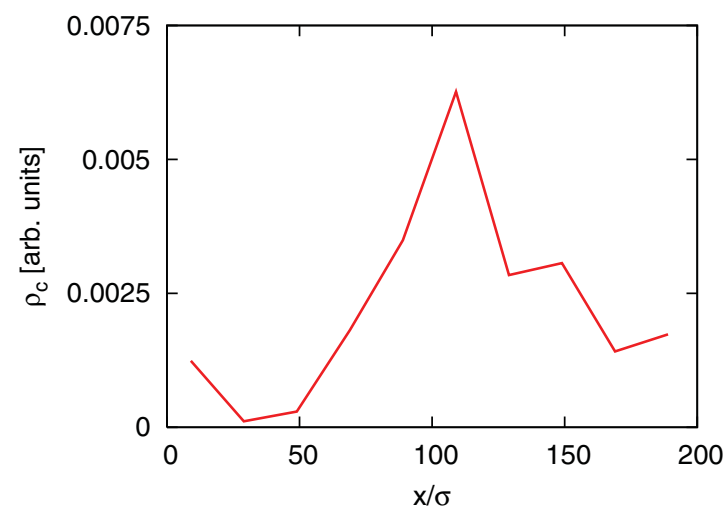

FIG. 10. (Color online) Colloid density profile along the channel corresponding to Fig. 9. 


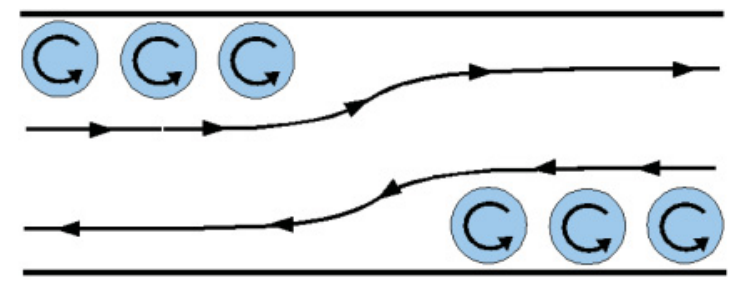

FIG. 11. (Color online) Illustration of the fluid flow caused by the fixed colloids in a channel segment with alternating pumping direction.

force as a function of $r$ shows a nonmonotonic behavior, in contrast to the narrowest channel.

\section{B. Ring channels with freely moving colloids}

We now consider several freely moving colloids in a ring channel. The applied external torque causes counterclockwise spinning of the colloids, as before. The curvature of the channel leads to a translational motion along the ring channel, which is quantified by the average tangential velocity of the colloids, shown in Fig. 13(a) for $D=1.5 \sigma, D=2 \sigma$ and $D=2.5 \sigma$ as a function of the medium channel radius $R$. A total counterclockwise motion in the annulus is observed in the simulations that monotonically decreases with increasing annulus diameter for all investigated channel widths. Over the investigated range of radii, the tangential velocity is well described by a power law:

$$
v_{\tan } \propto R^{-\gamma} .
$$

Due to the complex interplay of geometric and hydrodynamic effects, the exponent is not unique. For the largest torque $L_{\text {ext }, 0}=150 k_{B} T$, we find $\gamma \approx 1.7$ for the smallest channel width, $D=1.5, \gamma \approx 0.76$ for $D=2.0$, and $\gamma \approx 1.0$ in wider channels, $D=2.5$ and 3.0 , where colloids can pass each other [14].

Figure 13(b) shows the corresponding results for the smallest torque $L_{\text {ext, } 0} / 9=16.7 k_{B} T$. Here, in the channel where the colloids can pass each other, with $D=2.5 \sigma$, we find the same exponent $\gamma \approx 1.0$. However, in narrower channels, the exponents are different from the case of large torque;

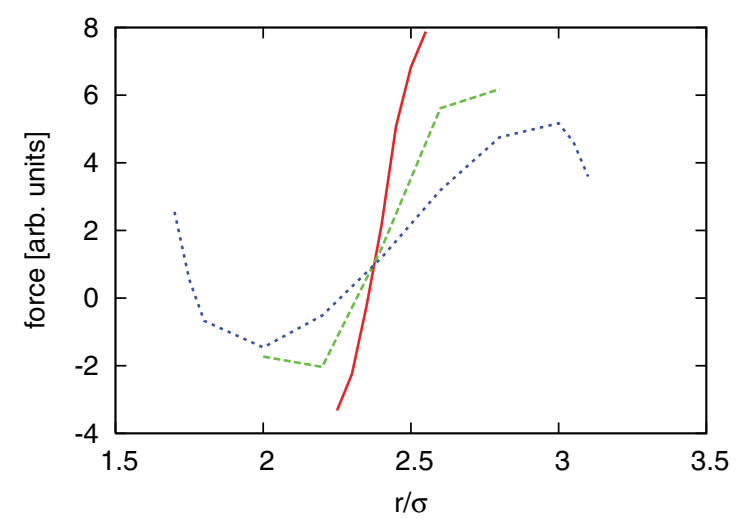

FIG. 12. (Color online) Tangential component of the force acting on a single rotating colloid with fixed axis at $r$ for $R=2.4 \sigma$ and $D=1.5 \sigma$ (red solid line), $2.5 \sigma$ (green dashed line), and $3.0 \sigma$ (blue dotted line).
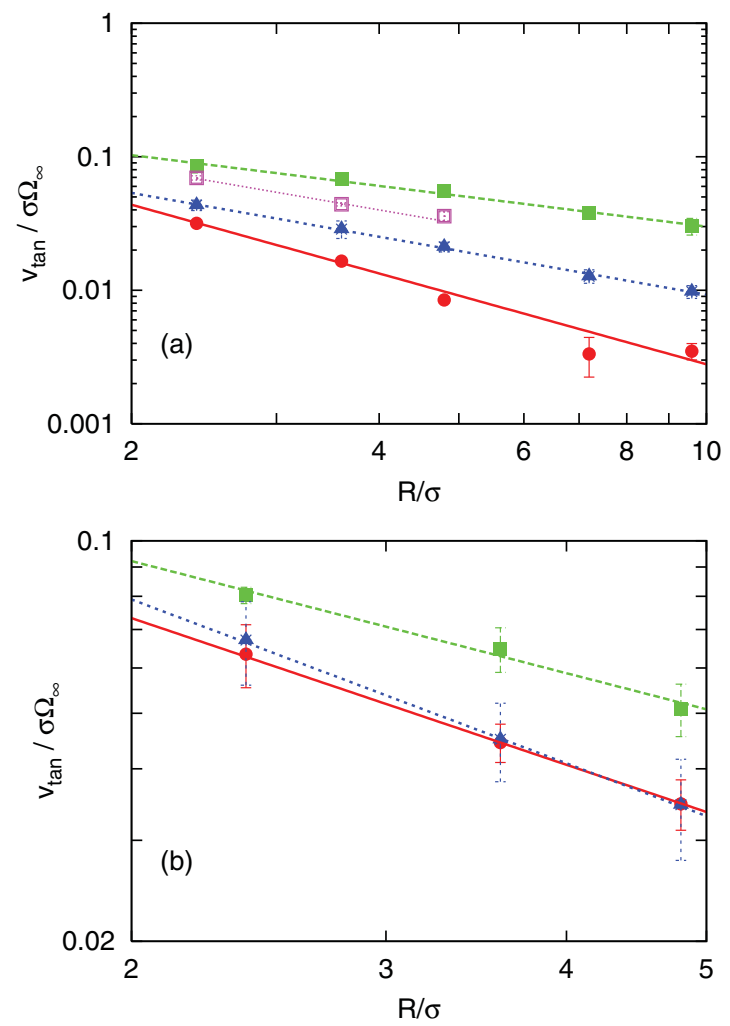

FIG. 13. (Color online) Average tangential velocity $v_{\tan }$ of spinning colloids as a function of the medium annulus radius $R$ for fixed channel widths $D=1.5 \sigma$ (red solid line), $2.0 \sigma$ (green dashed line), $2.5 \sigma$ (blue dotted line), and 3.0 $\sigma$ (magenta dashed-dotted line). The errors are estimated from four independent runs. (a) High Pe with torque $L_{0}^{\text {ext }}$ and (b) low Pe with torque $L_{0}^{\text {ext }} / 9$.

we now obtain $\gamma \approx 0.85$ for $D=1.5 \sigma$, and $\gamma \approx 0.65$ for $D=2.0 \sigma$. We thus conclude that the exponent for wide channels, where colloids can pass each other, is universal, $\gamma \approx 1.0$, independent of the channel width and the Péclet number (at least for $\mathrm{Pe}>1$ ), while the effective exponent for narrow channels is nonuniversal. In particular for the very narrow channel, the effective exponent depends very sensitively on Pe.

When the channel width is varied for a fixed medium radius, the tangential velocity shows an interesting, nonmonotonic behavior. We consider channel widths from $D=1.5 \sigma$ to $3.0 \sigma$ for fixed medium radii $R=2.4 \sigma, R=3.6 \sigma$, and $R=4.8 \sigma$ (see Fig. 14). In the narrowest channels, where colloids cannot pass each other, the colloids tend to stay close to the outer wall [see density distribution in Fig. 15(a) and snapshot in Fig. 16(a)]. This configuration allows a nearly unhindered circulation of the fluid around the inner confining cylinder in the opposite direction (compared to the motion of the colloids); see Fig. 15(b). Hence, the average tangential velocity is small. The strong confinement in the narrow channel leads to a reduced spinning angular velocity compared to wide channels.

A maximum in the tangential velocity is reached in channels with $D \approx 2.0 \sigma$. Here, because of the additional repulsive Lennard-Jones potential, two colloid just cannot pass each other. Due to the mutual forces acting on colloids close 


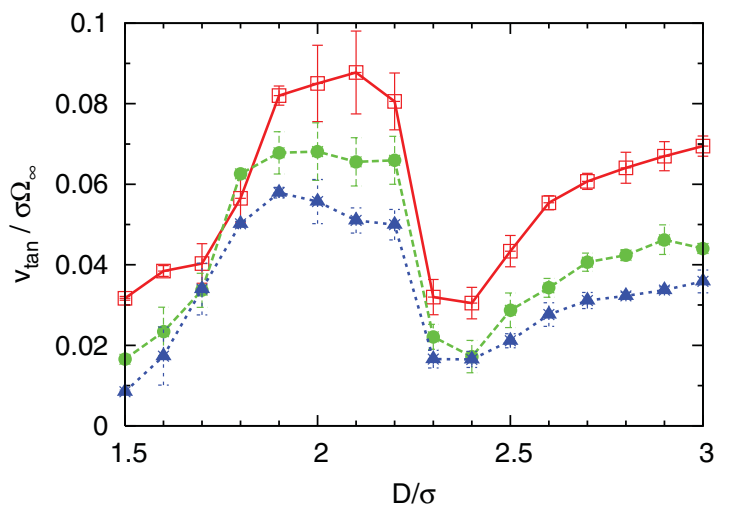

FIG. 14. (Color online) Average tangential velocity $v_{\tan }$ of the colloids as a function of channel widths $D$ for fixed annulus radii $R=$ $2.4 \sigma$ (red solid line), $R=3.6 \sigma$ (green dashed line), and $R=4.8 \sigma$ (blue dotted line). The errors are estimated from four independent runs.

to the inner and and outer walls, pairs of colloids become wedged together into the channel [see snapshot in Fig. 16(b) for $R=2.4$ and density profile in Fig. 15(a)]. In this way, the colloids are forming plugs that the fluid can hardly pass, and the fluid is efficiently dragged along with these pairs, and a strong backflow of the fluid that would hinder the motion of the colloids is prevented, as demonstrated by the
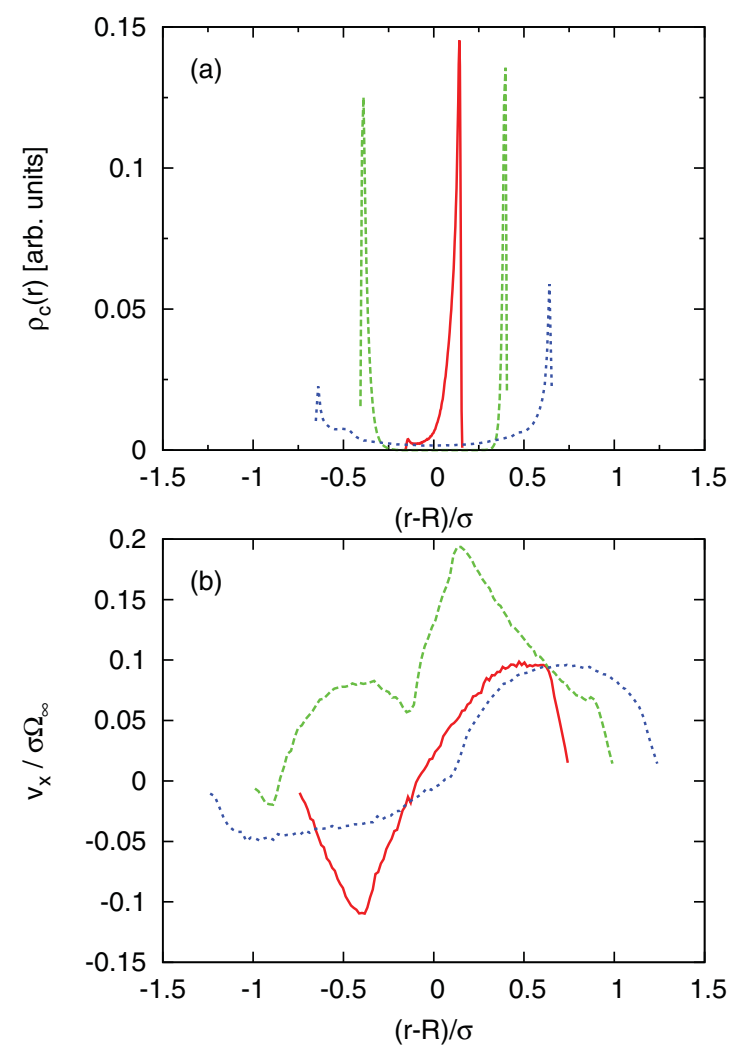

FIG. 15. (Color online) (a) Radial colloid density and (b) tangential fluid velocity for $R=2.4 \sigma$ and $D=1.5 \sigma$ (red solid line), $D=2.0 \sigma$ (green dashed line), and $D=2.5 \sigma$ (blue dotted line). The width of the curves in (a) and (b) is different, because colloids cannot approach the walls closer than $\sigma / 2+\delta_{\mathrm{LJ}}$.
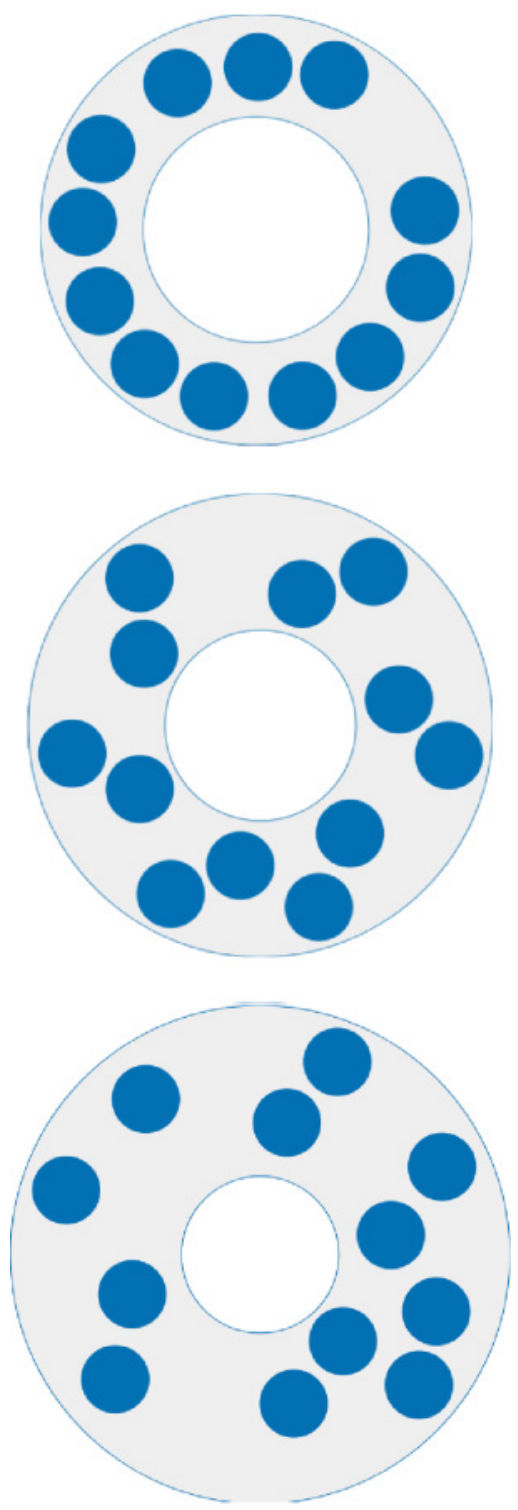

FIG. 16. (Color online) Snapshots of rotating colloids in ring channels with median radius $R=2.4 \sigma$, and widths $D=1.5 \sigma, 2.0 \sigma$, and $2.5 \sigma$ (top to bottom). Movies of the colloid motion in ring channels are provided in the supplemental material [52].

tangential fluid velocity profile in Fig. 15(b), which is positive (counterclockwise motion) over essentially the whole cross section of the channel. A maximum of the tangential velocity is also observed for $R=3.6 \sigma$ and $R=4.8 \sigma$. Here, although triplets can be observed transitionally, pairs of colloids are the preferred configuration.

The observation that the pairs move counterclockwise is consistent with the forces measured on single rotating colloids fixed at different radii (compare Sec. IV A). Here the forces acting on colloids close to the inner and outer walls point in opposite directions, but the magnitude of the force acting on the colloids near the outer wall is larger than on the colloids near the inner wall. Hence, a net force resulting in motion in the counterclockwise direction is expected. However, the presence of other colloids influences the velocity field and thereby also 


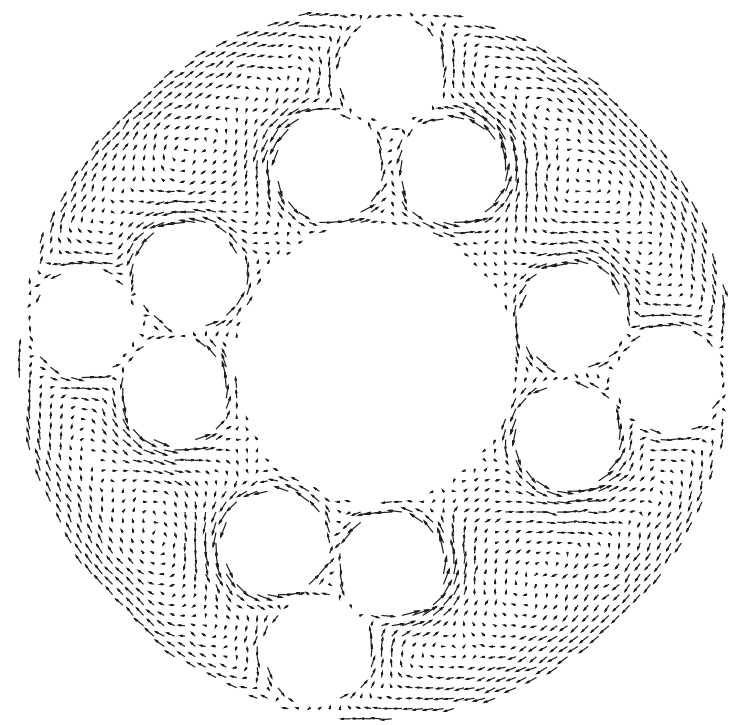

FIG. 17. Fluid velocity field, measured in a corotating frame, for ring channels with $R=2.4 \sigma$ and $D=2.1 \sigma$.

the forces acting on a single colloid, so taking the sum of the forces can be only a first approximation.

In the range of $D=2.0 \sigma$ to $2.2 \sigma$, we observe the formation of colloid triplets as well as pairs and combination of both, depending on the initial conditions (sequence of random numbers), where both are stable for roughly 500 full circulations $\left(2 \times 10^{7}\right.$ MPC steps). Since all involved interactions are purely repulsive, the stabilization of the clusters must be due to hydrodynamics. The highest tangential velocity results for triplets, which are observed more often for small $R$. The corresponding fluid velocity field, measured in a corotating frame, is shown in Fig. 17. Here fluid vortices (with clockwise circulation) appear between neighboring colloidal triplets, which stabilize the colloid clusters.

In wider channels, with $D>2.2 \sigma$, where two colloids can pass each other, the colloids at the inner wall move in the opposite direction compared to those at the outer wall, thereby reducing the overall tangential velocity [see snapshot Fig. 16(c)]. Figure 15(b) shows for $D=2.5 \sigma$ that there is a significant backflow near the inner wall, hence the overall fluid

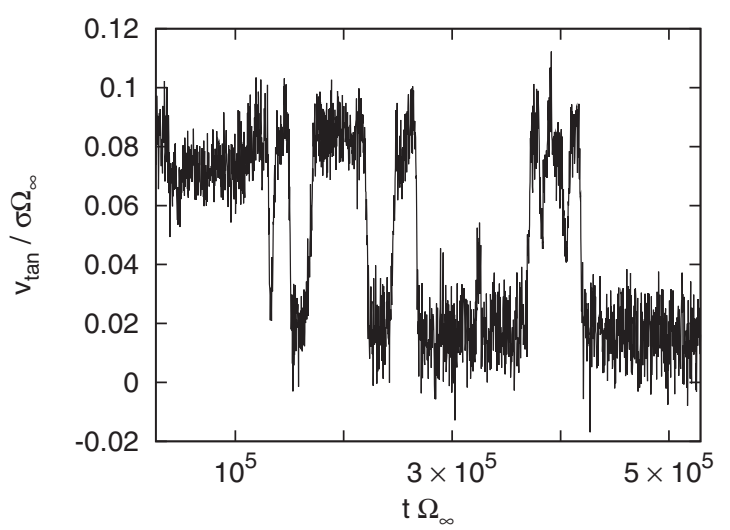

FIG. 18. Tangential colloid velocity as a function of time $t$ (scaled with the spinning frequency $\Omega_{\infty}$ in a bulk fluid) for $R=2.4 \sigma$ and $D=1.7 \sigma$.
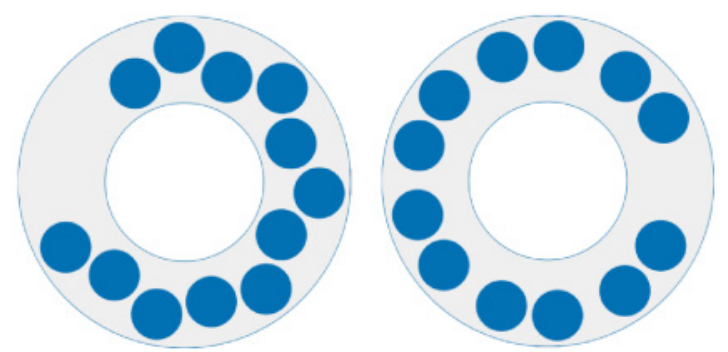

FIG. 19. (Color online) Snapshots of the two different states for $R=2.4 \sigma$ and $D=1.7 \sigma$. In the zigzag-like state (left), the colloids move faster than in the aligned state (right). A movie of the colloid motion in this channels is provided in the supplemental material [52].

transport is diminished. Increasing the channel width further, the tangential velocity rises again. This might be a similar effect as in the case of straight channels, where the average velocity per half channel is higher for broader channels. Since we keep the colloid line density fixed, the volume density decreases with increasing $D$. Moreover, due to geometric reasons, there are more colloids near the outer wall than near the inner wall.

\section{Velocity fluctuations in narrow ring channels}

For intermediate channel widths $(D=1.6 \sigma$ to $1.8 \sigma)$, the system shows an interesting dynamical behavior. The continous increase of the average colloid velocity shown in Fig. 14 hides the fact that the system can alternate between two different states with two distinct velocities, see Fig. 18, a zig-zag phase, where backflow is hindered by blocking colloids, resulting in a fast motion, and an aligned phase, where all colloids have the same radial distance from the center of the channel ring (Fig. 19). The average tangential velocities in the two states differ strongly, by a factor 4 , resulting in large variations for the average tangential velocity in Fig. 14. The transition between two different states for $R=2.4 \sigma$ and $D=1.7 \sigma$ is quite abrupt as shown in Fig. 18. When the leading colloid sheers out of line due to thermal fluctuations, fluid is pumped into the resulting gap, destroying the order of the subsequent colloids. Figure 18 shows the tangential colloid velocity as a function of time, clearly showing the two distinct states. A similar behavior is observed for $D=1.6$ and 1.8.

\section{SUMMARY AND CONCLUSIONS}

We have investigated spinning colloids in confined geometries - linear and ring channels — by a mesoscale hydrodynamics simulation technique that naturally includes thermal fluctuations. We focus on a concentration regime, where hydrodynamic interactions, volume exclusion, and thermal fluctuations dominate the dynamical behavior.

In linear channels, with channel diameters somewhat larger than two the colloid diameters, the colloids tend to stay close to the walls at which they move in opposite directions; due to symmetry reasons, there is no overall flow. When the twofold rotational symmetry of the channel is broken, net flow can be obtained. This can be achieved by fixing the positions of a periodic array of colloids near one of the two walls. In this way the flow direction can not only be controlled but also switched 
externally by changing the location of the fixed colloids. In an experiment, the position of a selected number of colloids can be fixed, for example, by laser tweezers $[53,54]$. It seems possible to use this approach to control the flow of complex fluids in complex microchannel networks.

Rotating electrical fields to generate flow in water-filled nanotubes (with the elctrical dipole of the water molecules coupling to the electrical field) have been suggested and investigated recently in Refs. [15,16]; here symmetry breaking and net flow in a preferred direction are achieved by different boundary conditions (slip and no-slip) at opposing walls. An advantage of our approach employing magnetic colloids is that it offers more flexibility for dynamically changing the flow direction and magnitude in microfluidic networks.

Transport velocities and Péclet numbers in typical microfluidic systems can be estimated from the results of Sec. III. With the spinning frequency $\Omega=125 \mathrm{~Hz}$ of the experimental system of Ref. [3], and the scaled half-channel velocity $v_{x} / \sigma \Omega \simeq 0.05$ from Fig. 3, we obtain for colloidal particles in water with a diameter $\sigma=10 \mu \mathrm{m}$ velocities $v_{x}$ in the range of 50 to $100 \mu \mathrm{m} / \mathrm{s}$ and Péclet numbers Pe $=3 \pi \eta \sigma^{2} v_{x} /\left(k_{B} T\right)$ of about $10^{4}$. However, there is a strong size dependence of the Péclet number on the colloid diameter, $\mathrm{Pe} \sim \sigma^{3}$, so that for smaller colloids of diameter $\sigma=1 \mu \mathrm{m}$, we predict $v_{x} \simeq 1 \mu \mathrm{m} / \mathrm{s}$ and $\mathrm{Pe} \simeq 10$. Therefore, thermal fluctuations are clearly relevant in systems of smaller colloids.

In circular channels, the two-way-traffic-like behavior that is already observed in linear channels is superimposed with a net flow induced by the channel curvature. Due to the complex interplay of geometric and hydrodynamic effects, the resulting average colloid velocity in the channel shows a complex behavior as a function of median radius, channel width, and spinning frequency. Moreover, hydrodynamically induced self-organization of the purely repulsive colloids is found. The most efficient propulsion is achieved for channel widths, when two colloids just cannot pass each other and thus block their converse motion. Here pairs of colloids form plugs that efficiently push the fluid column ahead.

\section{ACKNOWLEDGMENTS}

We thank R. G. Winkler (Jülich) and C. Bechinger (Stuttgart) for stimulating discussions. Financial support by the EU Collaborative Research Project "NanoDirect" (NMP4SL-2008-213948) is gratefully acknowledged.
[1] B. A. Grzybowski, H. A. Stone, and G. M. Whitesides, Nature (London) 405, 1033 (2000).

[2] B. A. Grzybowski and G. M. Whitesides, Science 296, 718 (2002).

[3] S. Bleil, D. W. M. Marr, and C. Bechinger, Appl. Phys. Lett. 88, 263515 (2006).

[4] B. Kavčič, D. Babič, N. Osterman, B. Podobnik, and I. Poberaj, Appl. Phys. Lett. 95, 023504 (2009).

[5] N. Elsner, C. P. Royall, B. Vincent, and D. R. E. Snoswell, J. Chem. Phys. 130, 154901 (2009).

[6] A. Terray, J. Oakey, and D. W. M. Marr, Science 296, 1841 (2002).

[7] K. Drescher, K. C. Leptos, I. Tuval, T. Ishikawa, T. J. Pedley, and R. E. Goldstein, Phys. Rev. Lett. 102, 168101 (2009).

[8] I. Llopis and I. Pagonabarraga, Eur. Phys. J. E 26, 103 (2008).

[9] R. Daw and J. Finkelstein, Nature (London) 442, 367 (2006).

[10] P. D. Palma, P. Valentini, and M. Napolitano, Phys. Fluids 18, 027103 (2006).

[11] A. Malevanets and R. Kapral, J. Chem. Phys. 110, 8605 (1999).

[12] R. Kapral, Adv. Chem. Phys. 140, 89 (2008).

[13] G. Gompper, T. Ihle, D. M. Kroll, and R. G. Winkler, Adv. Polym. Sci. 221, 1 (2009).

[14] I. O. Götze and G. Gompper, EPL 92, 64003 (2010).

[15] D. J. Bonthuis, D. Horinek, L. Bocquet, and R. R. Netz, Phys. Rev. Lett. 103, 144503 (2009).

[16] D. J. Bonthuis, D. Horinek, L. Bocquet, and R. R. Netz, Langmuir 26, 12614 (2010).

[17] G. G. Stokes, Trans. Cambridge Phil. Soc. 9, 8 (1851).

[18] G. B. Jeffery, Proc. R. Soc. London A 101, 169 (1922).

[19] E. J. Watson, Mathematika 42, 105 (1995).

[20] M. C. Sharatchandra, M. Sen, and M. G. el Hak, J. Heat Transf. 120, 99 (1998).
[21] D. DeCourtye, M. Sen, and M. Gad-El-Hak, Int. J. Comput. Fluid D 10, 13 (1998).

[22] H. Juárez, R. Scott, R. Metcalfe, and B. Bagheri, Comput. Fluids 39, 547 (2000).

[23] A. A. Mammoli and M. S. Ingber, Int. J. Numer. Meth. Eng. 44, 897 (1999).

[24] M. Hajjam, Z. Angew. Math. Phys. 49, 934 (1998).

[25] W. W. Hackborn, Can. Appl. Math. Q. 8, 171 (2000).

[26] J. T. Padding and A. A. Louis, Phys. Rev. Lett. 93, 220601 (2004).

[27] J. T. Padding and A. A. Louis, Phys. Rev. E 74, 031402 (2006).

[28] M. Hecht, J. Harting, T. Ihle, and H. J. Herrmann, Phys. Rev. E 72, 011408 (2005).

[29] J. Sané, J. T. Padding, and A. A. Louis, Phys. Rev. E 79, 051402 (2009)

[30] A. Malevanets and J. M. Yeomans, Europhys. Lett. 52, 231 (2000).

[31] M. Ripoll, K. Mussawisade, R. G. Winkler, and G. Gompper, Europhys. Lett. 68, 106 (2004).

[32] M. Ripoll, R. G. Winkler, and G. Gompper, Phys. Rev. Lett. 96, 188302 (2006).

[33] M. Ripoll, R. G. Winkler, and G. Gompper, Eur. Phys. J. E 23, 349 (2007).

[34] M. A. Webster and J. M. Yeomans, J. Chem. Phys. 122, 164903 (2005).

[35] S. H. Lee and R. Kapral, J. Chem. Phys. 124, 214901 (2006).

[36] N. Watari, M. Makino, N. Kikuchi, R. G. Larson, and M. Doi, J. Chem. Phys. 126, 094902 (2007).

[37] H. Noguchi and G. Gompper, Proc. Natl. Acad. Sci. USA 102, 14159 (2005).

[38] H. Noguchi and G. Gompper, J. Chem. Phys. 125, 164908 (2006). 
[39] T. Sakai, Y. Chen, and H. Ohashi, Phys. Rev. E 65, 031503 (2002).

[40] K. Tucci and R. Kapral, J. Chem. Phys. 120, 8262 (2004).

[41] H. Noguchi, N. Kikuchi, and G. Gompper, Europhys. Lett. 78, 10005 (2007).

[42] I. O. Götze, H. Noguchi, and G. Gompper, Phys. Rev. E 76, 046705 (2007).

[43] T. Ihle and D. M. Kroll, Phys. Rev. E 63, 020201(R) (2001).

[44] A. Lamura, G. Gompper, T. Ihle, and D. M. Kroll, Europhys. Lett. 56, 319 (2001).

[45] H. Noguchi and G. Gompper, Phys. Rev. E 78, 016706 (2008).

[46] W. Müller, Ing.-Arch. 13, 37 (1942).

[47] D. J. Jeffrey and Y. Onishi, Q. J. Mech. Appl. Math. 34, 129 (1981).

[48] M. Sen, D. Wajerski, and M. Gad-El-Hak, J. Fluids Eng. 118, 624 (1996).
[49] Y. Ueda, A. Seller, T. Kida, and M. Nakanishi, J. Fluid Mech. 495, 225 (2003).

[50] G. Segre and A. Silberberg, Nature (London) 189, 209 (1961).

[51] S. Redner, A Guide to First-Passage Processes (Cambridge University Press, New York, 2001).

[52] See Supplemental Material at http://link.aps.org/supplemental/ 10.1103/PhysRevE.84.031404 for simulation animations of the motion of spinning colloids in linear and ring channels; ring channels with three different channel widths are considered, as well as a linear channel with one colloid position fixed near the wall.

[53] E. R. Dufresne and D. G. Grier, Rev. Sci. Instrum. 69, 1974 (1998).

[54] J. P. Hoogenboom, D. L. J. Vossen, C. Faivre-Moskalenko, M. Dogterom, and A. van Blaaderen, Appl. Phys. Lett. 80, 4828 (2002). 